\title{
Third mobile window associated with suspected otosclerotic foci in two patients with an air-bone gap
}

\author{
V VAN ROMPAEY ${ }^{1}$, J POTVIN $^{1}$, L VAN DEN HAUWE $^{2}$, P VAN DE HEYNING $^{1}$ \\ ${ }^{1}$ Departments of Otorhinolaryngology and Head and Neck Surgery, and ${ }^{2}$ Radiology, Antwerp University Hospital, \\ Edegem, Belgium
}

\begin{abstract}
Objective: To demonstrate the need for computed tomography imaging of the temporal bone in patients clinically suspected of otosclerosis who present with atypical symptoms or audiological findings.

Case reports: We present two patients with bilateral conductive hearing loss and suspected otosclerosis in whom third mobile window lesions were revealed. The first patient had bilateral large vestibular aqueducts and bilateral fenestral otosclerotic foci. Computed tomography imaging of the second case revealed bilateral superior semicircular canal dehiscence and bilateral cochlear clefts, mimicking an otosclerotic focus in the fissula ante fenestram.

Conclusion: Differentiating third mobile window lesions from otosclerosis as the cause of a conductive hearing loss is essential before considering stapes surgery, as such treatment would be unnecessary and potentially harmful.
\end{abstract}

Key words: Otosclerosis; Vestibular Aqueduct; Conductive Hearing Loss; Computed Tomography; X-Ray

\section{Introduction}

Otosclerosis is a disease characterised by a progressive focal dysplasia of the enchondral bone of the labyrinth capsule, causing a progressive unilateral or bilateral conductive hearing loss. ${ }^{1}$ Otosclerosis is a clinical and audiometric diagnosis. A computed tomography (CT) scan of the temporal bone is advisable to screen for third mobile window lesions causing a conductive hearing loss of inner ear origin. This can be suspected if an air-bone gap (ABG) is accompanied by normal tympanometry and present stapedial tendon reflexes, or if an ABG is accompanied by absent stapedial tendon reflexes associated with other findings not typical of otosclerosis (e.g. vertigo or autophony). ${ }^{2-4}$

We present two cases with bilateral conductive hearing loss which were highly suspicious for otosclerosis. Computed tomography imaging of the first case demonstrated bilateral large vestibular aqueducts and bilateral fenestral otosclerotic foci. Computed tomography imaging of the second case revealed bilateral superior semicircular canal dehiscence and bilateral cochlear clefts, mimicking an otosclerotic focus in the fissula ante fenestram.

\section{Case reports}

Case one

A 33-year-old woman presented with bilateral hearing loss, autophony and continuous non-pulsatile tinnitus. Vertigo or equilibrium disorders were not reported. Her family history included Ménière's disease and otosclerosis.

Microscopic examination of the eardrum was normal.
Tympanometry showed bilateral type A curves with absent stapedial tendon reflexes. Pure tone audiometry revealed bilateral conductive hearing loss with a Carhart notch at $2 \mathrm{kHz}$ and supranormal bone conduction thresholds at lower frequencies (Figure 1). Although a Carhart notch was present and stapedial tendon reflexes were absent, the supranormal bone conduction was not typical of otosclerosis.

We performed a CT scan of the temporal bone to exclude third window lesions. The bony vestibular aqueduct diameter, measured in the middle between the crus and the external operculum, was larger than the posterior semicircular canal transverse diameter. The bony vestibular aqueduct diameter measured 1.9 and $1.55 \mathrm{~mm}$ on the right and left side, respectively, which is more than the $1.5 \mathrm{~mm}$ required for the diagnosis of a large vestibular aqueduct. ${ }^{5,6}$ Hypoattenuation was seen in the pericochlear and anterior oval window region. Based on these findings, this patient was diagnosed with large vestibular aqueducts in conjunction with bilateral fenestral and retrofenestral otosclerosis (Figure 2).

In this patient's case, we opted for conservative treatment rather than surgery.

\section{Case two}

A 52-year-old woman was referred to us with bilateral conductive hearing loss, more prominent on the right side. She did not report any vertigo or autophony. Her family history was unremarkable.

Microscopic examination of the eardrum was normal. 


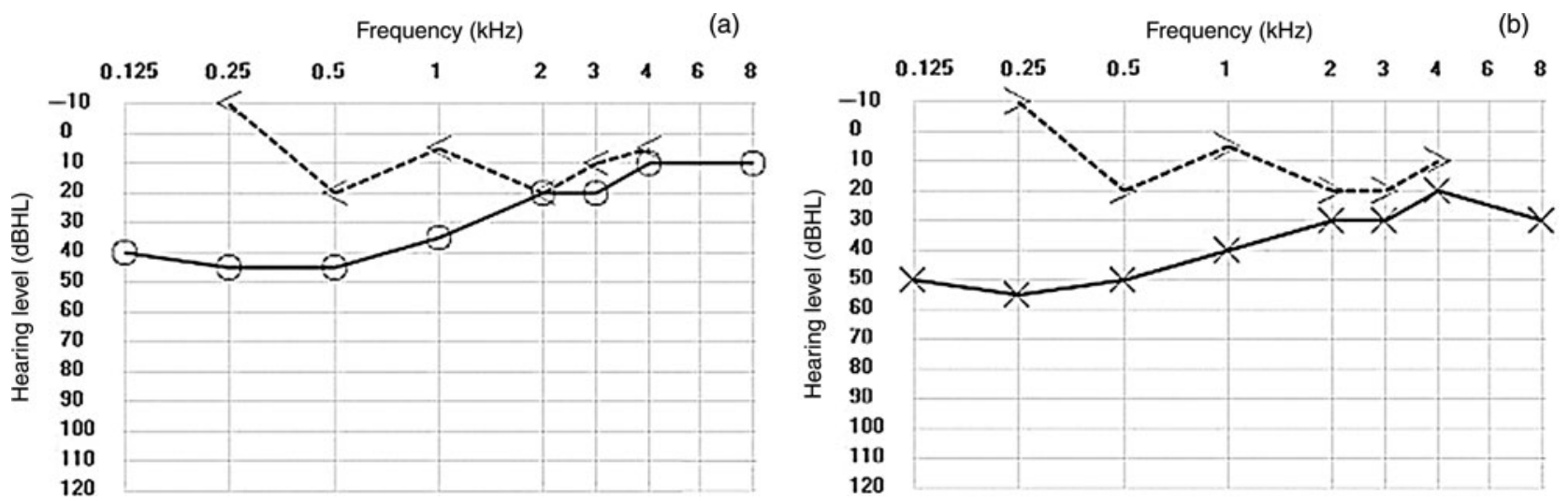

FIG. 1

Audiogram for patient one: (a) right ear, (b) left ear. A large air-bone gap is present on both sides at the low and middle frequencies. A Carhart notch is present at $2 \mathrm{kHz}$. Supranormal bone conduction thresholds are found bilaterally at $250 \mathrm{~Hz}$. < = right masked bone conduction thresholds; > = left masked bone conduction thresholds; $\times=$ left air conduction thresholds; $O=$ right air conduction thresholds

(a)

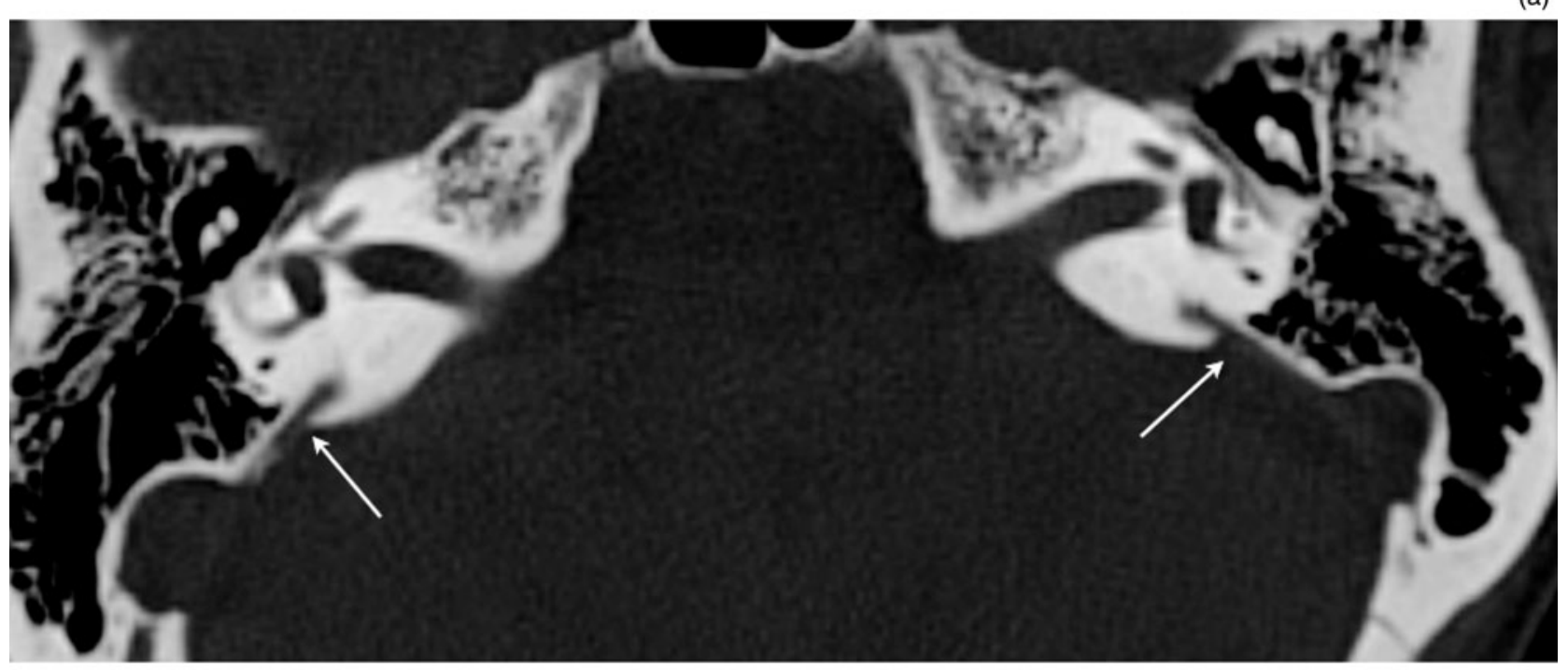

(b)

(c)
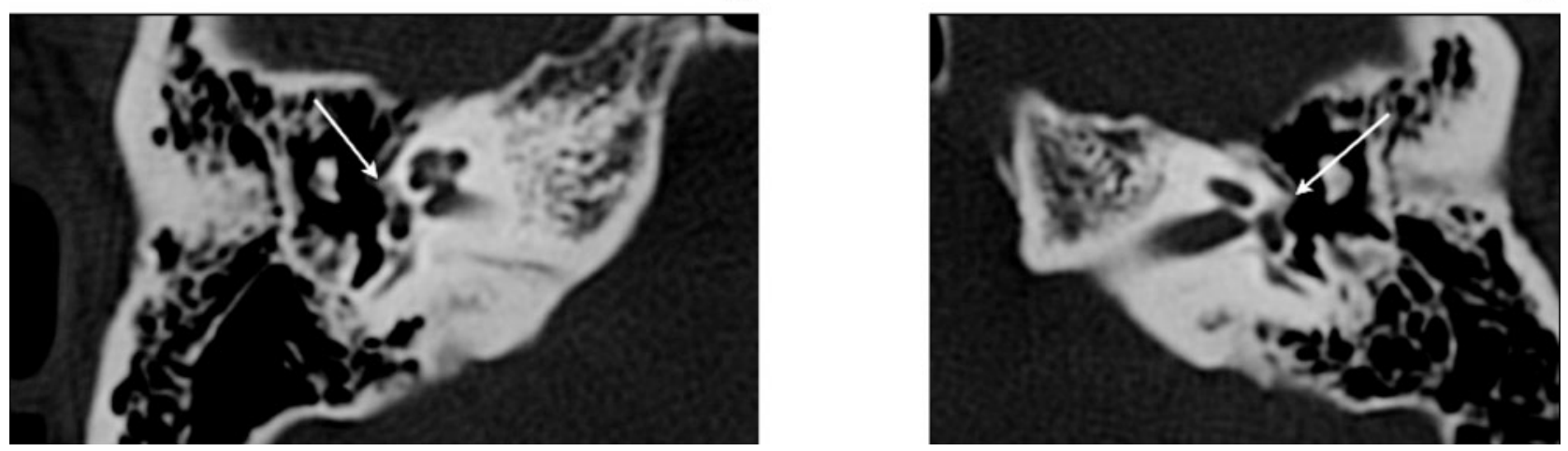

FIG. 2

Patient one: axial computed tomography scan showing bilateral large vestibular aqueducts (a) and bilateral otosclerotic foci at the fissula ante fenestram $(b$ and $c)$.

Tympanometry showed bilateral type A curves, with bilateral ipsilateral and contralateral stapedial tendon reflexes. Pure tone audiometry revealed a bilateral conductive hearing loss (more prominent on the right side) with supranormal bone conduction thresholds at lower frequencies (Figure 3).

A diagnosis of an otospongiotic focus at the fissula ante fenestram was proposed by the referring ENT physician 

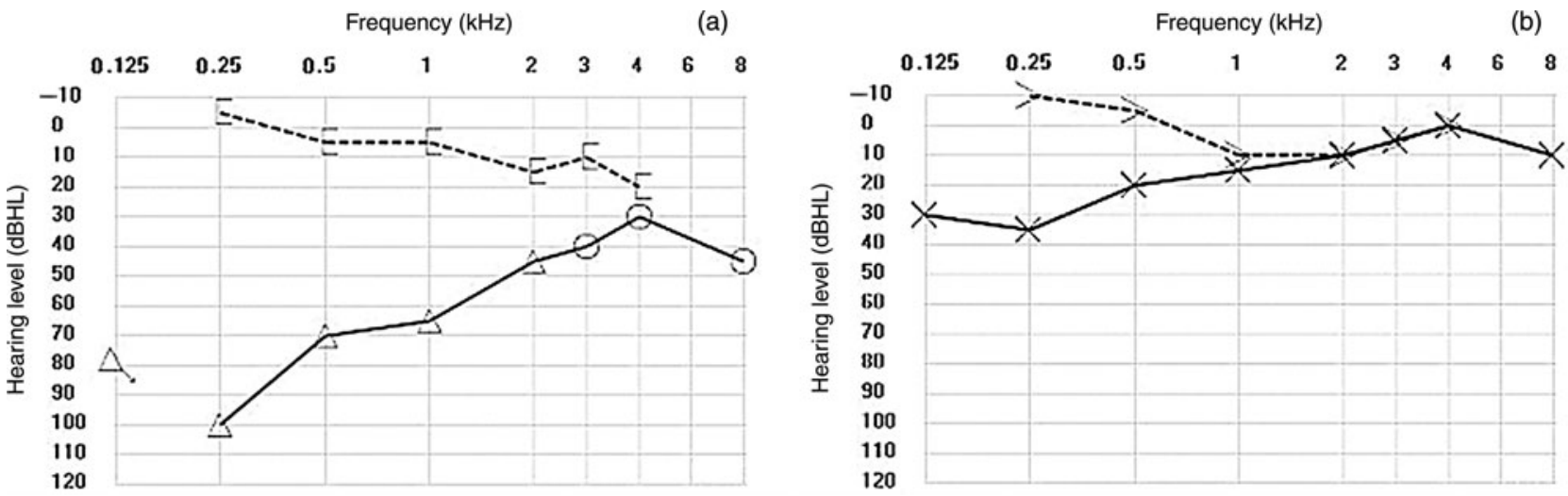

FIG. 3

Audiogram for patient two: (a) right ear, (b) left ear. A large air-bone gap is present on both sides at the low and middle frequencies, more pronounced on the right side. The air-bone gap disappears at $2 \mathrm{kHz}$ on the left side, but remains present at all frequencies on the right side. Supranormal bone conduction thresholds are found bilaterally at $250 \mathrm{~Hz}$. [ = right masked bone conduction thresholds; > = left masked bone conduction thresholds; $x=$ left air conduction thresholds; $O=$ right air conduction thresholds; $\Delta=$ right masked air conduction thresholds

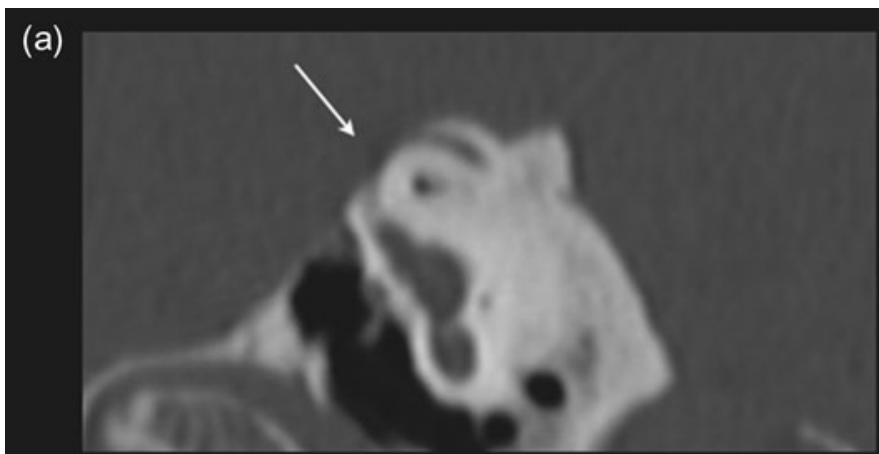

(b)
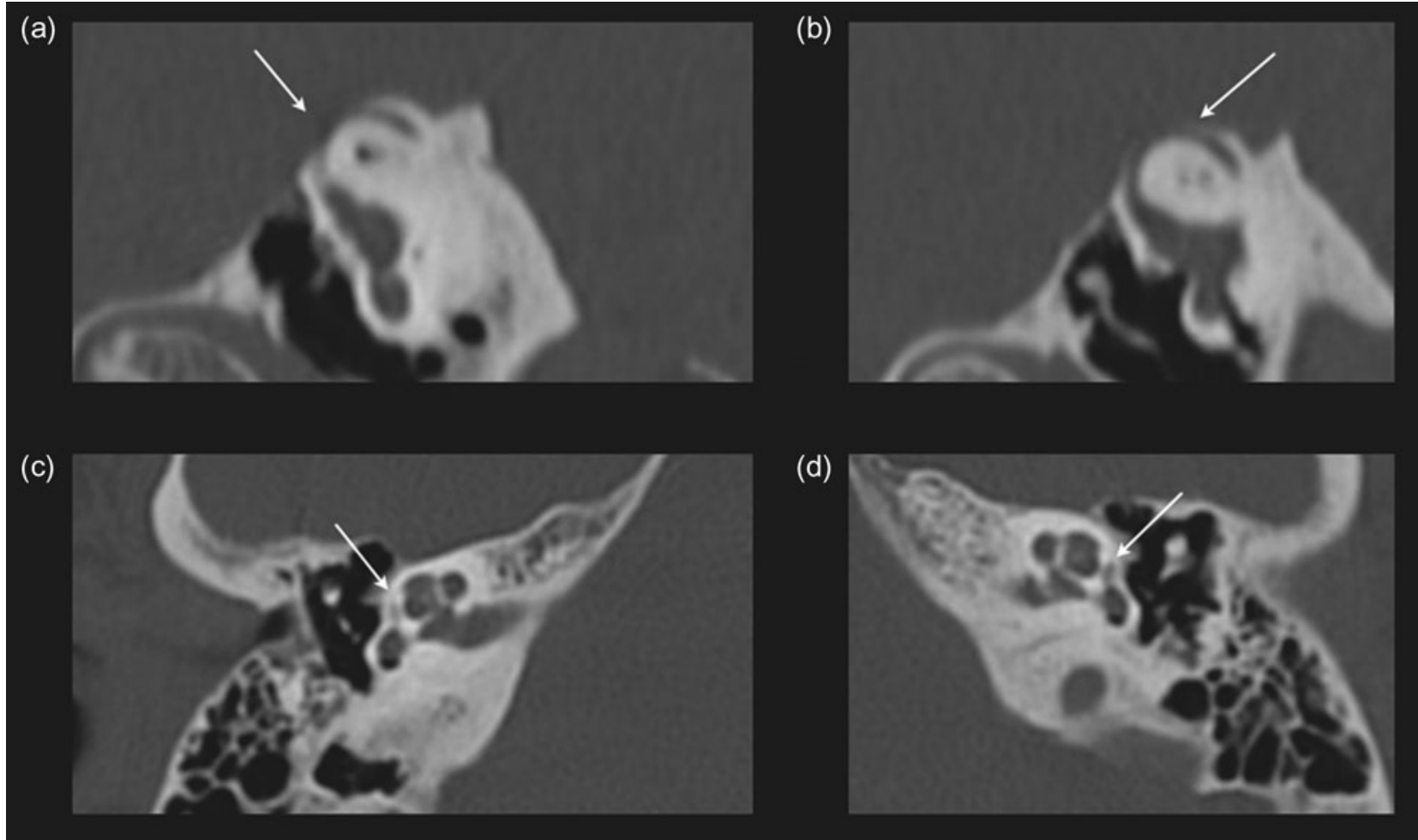

(d)

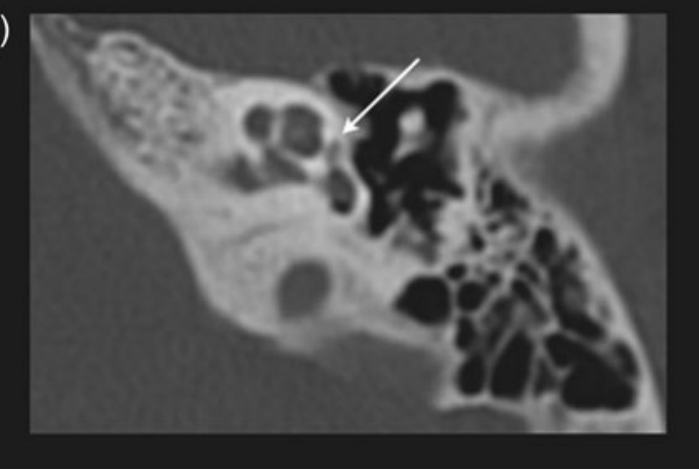

FIG. 4

Patient two: axial computed tomography scan showing bilateral superior semicircular canal dehiscence (a and b), and bilateral cochlear clefts starting at the fissula ante fenestram and continuing along the basal turn of the cochlea (c and d).

and radiologist, based on a CT scan of the temporal bone. On closer inspection of the same images, we also suspected bilateral superior semicircular canal dehiscence. We repeated the $\mathrm{CT}$ scan due to suboptimal quality, confirming the diagnosis of an extensive superior semicircular canal dehiscence on the right side and a less severe variant on the left side. When studying the fissula ante fenestram in detail, we could find no otospongiotic focus. We did find a so-called 'cochlear cleft' of the otic capsule (Figure 4).

Tone-burst cervical vestibular evoked myogenic potentials were present and revealed a lowered threshold and high amplitudes, ruling out ossicular fixation, a middle-ear process or saccular loss, and confirming superior semicircular canal dehiscence as the most probable aetiology of the conductive hearing loss. ${ }^{7}$

In this patient, we also opted for conservative treatment, suggesting a hearing aid on the right side.

\section{Discussion}

The clinical and audiometric presentation of a large vestibular aqueduct or superior semicircular canal dehiscence is known to mimic that of otosclerosis due to a phenomenon termed 'conductive hearing loss of inner ear origin'. ${ }^{2}$ In 
atypical cases of otosclerosis, the diagnosis can be made by performing high-resolution CT scanning with reformatting of the images in planes parallel and perpendicular to that of the superior semicircular canal.

To our knowledge, our first patient's CT scan represents the first imaging report of bilateral otosclerotic foci combined with bilateral large vestibular aqueducts. One similar temporal bone histology case has been published. ${ }^{8}$

- Temporal bone computed tomography is useful to screen for third mobile window lesions in patients with suggested otosclerosis but with atypical symptoms or present stapedial tendon reflexes

- This paper describes the first reported patient with bilateral otosclerotic foci and bilateral large vestibular aqueducts

- The authors stress the clinical importance of distinguishing a cochlear cleft from an otosclerotic focus in the fissula ante fenestram

We decided not to perform a stapedotomy in this patient for several reasons. There was a possibility that the conductive hearing loss was in fact a conductive hearing loss of inner ear origin caused by the large vestibular aqueduct. ${ }^{2,9}$ This could mean that the hearing loss would not respond to stapedotomy. Because the otosclerotic focus reduces the mobility of the round window, a stapedotomy could create a third window, worsening the hearing loss. There was also an increased risk of a stapes 'gusher' during the procedure.

Our second patient's hearing loss was probably caused by her superior semicircular canal dehiscence and not by the presumed otosclerotic focus, which was in fact a cochlear cleft. The presence of a cochlear cleft that mimics an otosclerotic focus on CT scan is less well known, and should also be considered in the differential diagnosis when audiometric findings do not agree or other radiological manifestations potentially indicate a conductive hearing loss of inner ear origin. A cochlear cleft is a small, nonosseous space occupying a short segment of a spiral plane in the middle of the otic capsule, parallel to the basal turn of the cochlea. The cochlear cleft is a radiological finding and is frequently seen (in up to 41 per cent) in magnified temporal bone CT images in children. It is found less often with advancing age. ${ }^{10}$

The importance of distinguishing these lesions from an otosclerotic focus lies in the fact that they preclude stapedotomy. No hearing improvement would occur, and surgery is thus unnecessary.

\section{References}

1 Mudry A. Adam Politzer (1835-1920) and the description of otosclerosis. Otol Neurotol 2006;27:276-81

2 Merchant SN, Nakajima HH, Halpin C, Nadol Jr. JBJr, Lee DJ, Innis WP et al. Clinical investigation and mechanism of air-bone gaps in large vestibular aqueduct syndrome. Ann Otol Rhinol Laryngol 2007;116:532-41

3 Merchant SN, Rosowski JJ. Conductive hearing loss caused by third-window lesions of the inner ear. Otol Neurotol 2008;29: 282-9

4 Minor LB. Clinical manifestations of superior semicircular canal dehiscence. Laryngoscope 2005;115:1717-27

5 Emmett JR. The large vestibular aqueduct syndrome. Am J Otol 1985;6:387-415

6 Govaerts PJ, Casselman J, Daemers K, De Ceulaer G, Somers T, Offeciers FE. Audiological findings in large vestibular aqueduct syndrome. Int J Pediatr Otorhinolaryngol 1999;51:157-64

7 Roditi RE, Eppsteiner RW, Sauter TB, Lee DJ. Cervical vestibular evoked myogenic potentials (cVEMPs) in patients with superior canal dehiscence syndrome (SCDS). Otolaryngol Head Neck Surg 2009;141:24-8

8 Ribeiro TK, Fayad JN. A large vestibular aqueduct syndrome associated with otosclerosis. Otol Neurotol 2008;29:725-6

9 Mikulec AA, McKenna MJ, Ramsey MJ, Rosowski JJ, Herrmann BS, Rauch SD et al. Superior semicircular canal dehiscence presenting as conductive hearing loss without vertigo. Otol Neurotol 2004;25:121-9

10 Chadwell JB, Halsted MJ, Choo DI, Greinwald JH, Benton C. The cochlear cleft. AJNR Am J Neuroradiol 2004;25:21-4

Address for correspondence:

Dr Vincent Van Rompaey,

Department of Otorhinolaryngology and Head and Neck Surgery,

University Hospital of Antwerp,

Wilrijkstraat 10 ,

2650 Edegem, Belgium

Fax: +3238254451

E-mail: vincent.vanrompaey@ua.ac.be

Dr P Van de Heyning takes responsibility for the integrity of the content of the paper

Competing interests: None declared 